\title{
Structural typologies of salivary calculi
}

P. Nolasco*, A.J. Anjos**, J.M. Aquino Marques**, F. Cabrita***, M.F.C. Pereira****, A.P. Alves de Matos $* * * * *$ and P.A. Carvalho*

*ICEMS, Instituto Superior Técnico, Universidade de Lisboa, Av. Rovisco Pais, 1049-001 Lisboa, Portugal

**Faculdade de Medicina Dentária, Universidade de Lisboa, Cidade Universitária, 1649-003 Lisboa, Portugal

***Serviço de Cirurgia Maxilo-Facial, Centro Hospitalar de Lisboa Central, R. José António Serrano 1150-199 Lisboa, Portugal

****CEGPIST, Departamento de Engenharia Civil, Arquitectura e Georrecursos, Instituto Superior Técnico, Universidade de Lisboa, Av. Rovisco Pais, 1049-001 Lisboa, Portugal

*****Anatomia Patológica, Centro Hospitalar de Lisboa Central - HCC, Rua da Beneficência 8, 1069-166 Lisboa; Centro de Estudos do Ambiente e do Mar (CESAM/FCUL) - Faculdade de Ciências da Universidade de Lisboa and Centro de Investigação Interdisciplinar Egas Moniz (CiiEM), Quinta da Granja , Monte de Caparica, 2829-511 Caparica, Portugal

Several theories have been put forward regarding the aetiology and pathogenesis of salivary calculi, although a comprehensive understanding of the nucleation and growth mechanisms involved in the formation of these structures is still lacking.

In general, sialoliths present one core partially or highly mineralized surrounded by concentric layers of organic and mineralized matter that alternate in succession following a chronologic sequence [1]. The layers consist of fine mineralized strata intercalated with fine organic ones and threaded globular structures with variable degrees of mineralization [1].

The exact mechanism involved in the genesis of sialoliths remains largely unknown, theories defending an initial organic nidus or an initial precipitation of minerals, with subsequent deposition of organic and inorganic layers, can be found in the literature [2-4]. Nevertheless, it remains object of discussion the etiologic factors responsible for the formation of the first nidus or the initial precipitation, since infection, inflammation of the gland, viscous nature of the mucous secretions or naturally existing sialomicroliths have all have been implicated [4-6].

Aiming at an exhaustive systematization of salivary calculi morphogenesis, their morphology has been studied by micro-computed tomography $(\mu \mathrm{CT})$ and scanning electron microscopy $(\mathrm{SEM}) . \mu \mathrm{CT}$ studies were done on asextracted dried samples using $\mu \mathrm{CT}$ SkyScan 1172 instrument with a 1.3 Megapixel camera, operated at the maximum available power of the source $(10 \mathrm{~W})$. Radiographs acquisition was performed with a rotational step in the $0.70-1^{\circ}$ range, until a maximum of $180^{\circ}$, with an exposure time in the 3.1-5 s range. Microscopy observations were carried out with backscattered electron (BSE) signals using a JEOL JSM 7001F operated at $15 \mathrm{kV}$, samples were previously prepared following metallographic procedures [1].

The submandibular and parotid calculi investigated presented similar growth patterns, which can follow either concentric (Figure 1) or perturbed-growth typologies (Figure 2), although in most situations a gradation between them has been found. Nevertheless, a single well-defined core constituted by material with low mineralization was frequently present, supporting the nucleation hypothesis of an initial organic nidus [7].

The combination of $\mu \mathrm{CT}$ with SEM enabled a comprehensive characterization of the sialoliths: (i) the former technique allowed for a precise localization of the core and other morphological features within the calculus volume, while (ii) investigation of details at higher resolution could be achieved with the latter method. However, due to the friable nature of the sialoliths, handling during sample preparation results often in material loss (compare (a) and (b) in both Figures). 
References

1. Alves de Matos A.P. et al., Microsc. and Microanal., 13:390, 2007.

2. Siddiqui S., Brit. Dent. J., 193:89, 2002.

3. Anneroth G. et al., Scand. J. Dent. Res., 86:182, 1978.

4. Teymoortash A. et al., Arch. Oral Biol., 48:233, 2003.

5. Grases F., Santiago C. et al., Clin. Chim. Acta, 334:131, 2003.

6. Harrison J.D., Otolaryng. Clin. N. Am., 42:927, 2009.

7. Sakae T. et al., J. Dent. Res., 60:842-844, 1981.

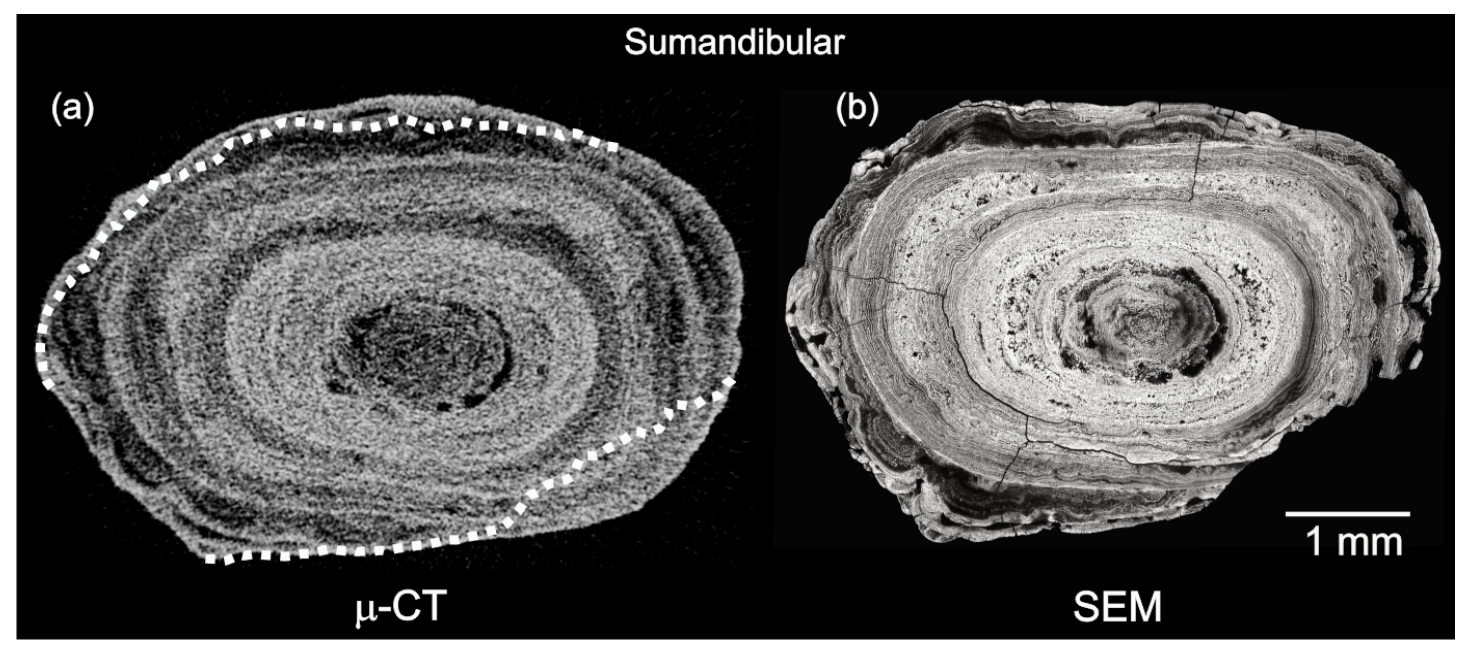

Figure 1. (a) $\mu-\mathrm{CT}$ and (b) SEM images of a submandibular calculus presenting an approximately concentric growth typology.

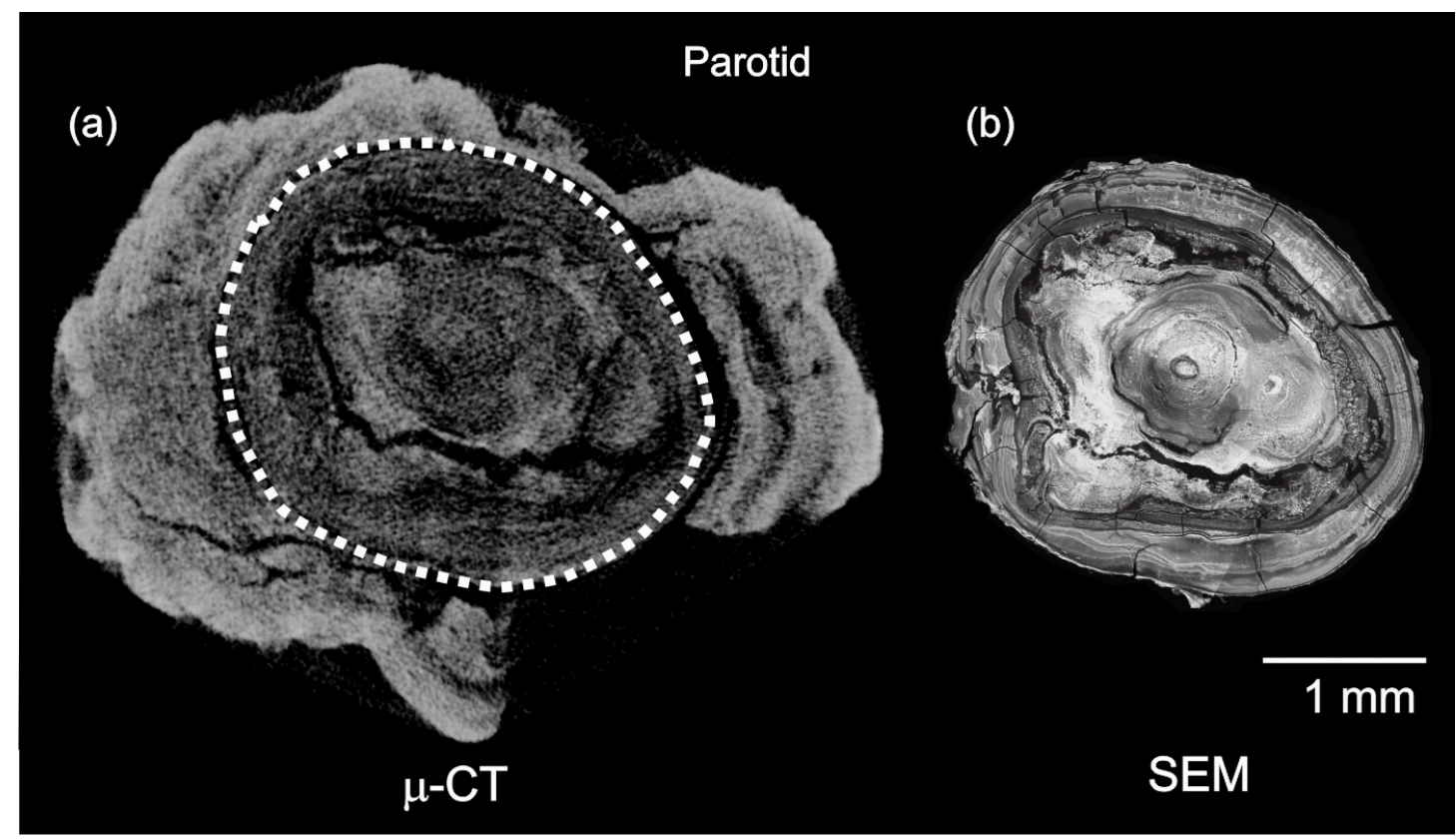

Figure 2. (a) $\mu$-CT and (b) SEM images of a parotid calculus presenting a perturbed-growth typology.

The work was carried out with financial support of the Portuguese Foundation for Science and Technology through PTDC/SAU-ENB/111941/2009 and PEst-OE/CTM-UI0084/2011 grants. 